\title{
Du tabou à la grammaire. Les sacres en français québécois dans une perspective interactionnelle
}

\author{
Valerie Bässler \\ Romanisches Seminar, Universität Freiburg \\ valerie.baessler@romanistik.uni-freiburg.de
}

\section{Introduction}

«Aï tabarnak, ça a fait mal ! ». Au Québec, cette formule souvent utilisée et légèrement modifiée selon le contexte, semble représenter la fonction principale d'un sacre, à savoir le défoulement. ${ }^{1}$ L'aspect psycholinguistique de ce phénomène langagier a déjà été formulé par Benveniste en 1974 ; selon ce linguiste, l'emploi des jurons ${ }^{2}$ revient à une simple décharge émotive : "Mais cette parole n'est pas communicative, elle est seulement expressive, bien qu'elle ait un sens. [...] Il [i.e. le juron] ne se réfère pas non plus au partenaire ni à une tierce personne. Il ne transmet aucun message, il n'ouvre pas de dialogue, il ne suscite pas de réponse. » (1974:256).

Dans cette étude, nous nous proposons de montrer que les sacres en français québécois représentent beaucoup plus que de simples jurons à base affective, qui servent uniquement à se défouler verbalement ${ }^{3}$, mais qu'ils peuvent aussi remplir des fonctions pragmatiques au sein de l'interaction. Ces analyses reposent sur un corpus établi en 2006, comprenant des genres discursifs différents des plus variés.

\section{Objet d'étude}

Les sacres existent probablement dans toutes les langues ou au moins dans la plupart d'entre elles : Sacrer semble être un besoin verbal des hommes leur permettant d'exprimer de l'affectivité langagière ainsi que de l'agression verbale. Ce qui est aussi commun aux langues, c'est le fait que chacune d'entre elles dispose de tabous et ce sont précisément ceux-ci qui sont touchés ou rompus par l'emploi des sacres. ${ }^{4}$

En français québécois, les sacres sont des jurons à base religieuse faisant référence à des objets liturgiques comme calvaire, tabernacle, hostie, ciboire ou calice mais vidés de leur sens originel. C'est la raison pour laquelle ils se distinguent nettement des jurons utilisés dans les autres langues romanes et germaniques qui quant à eux se réfèrent presque uniquement au domaine de la sexualité et de la scatologie (putain, bordel, joder, fuck, merde, mierda, Scheiße, shit, etc.). Dans toutes ces langues, cependant, les noms de Dieu et de saints sont utilisés dans des sacres ${ }^{5}$, mais de manière plus ou moins intense (mon Dieu, madonna, Gesù Cristo, Mein Gott, My God, Gosh, etc.).

Les sacres n'expriment pas seulement des émotions telles que la colère, la stupéfaction, la peur, l'envie, la reconnaissance, la joie etc., transportées à travers des interjections, mais de par leur lexicalisation poussée permettant un usage comme substantif, verbe, adjectif et adverbe, ils peuvent également remplir des fonctions grammaticales bien concrètes. ${ }^{6}$ Ce faisant, ils servent d'intensification ou de quantification et sont donc parfaitement intégrés dans la syntaxe de la langue parlée, et il n'est donc pas surprenant de voir, dans une grammaire parue au Québec ${ }^{7}$, un chapitre traitant les sacres de façon systématique et scientifique.

\section{Terminologie et catégorisation du vocabulaire religieux}

Les sacres sont un phénomène complexe parce qu'ils recouvrent plusieurs formes d'expression qui leur sont propres. La terminologie qu'on retrouve dans les ouvrages de référence varie selon les auteurs et leurs disciplines. ${ }^{8}$ Étant donné que ce travail prend en compte toutes les expressions émotives - peu importe leur 'degré'9 - et même si la notion suivante de sacre n'est pas partagée par bon nombre de Québécois, c'est faute d'une meilleure terminologie que nous entendons par sacres les jurons à base 
religieuse, ainsi que leurs dérivés (tabarouette), composés (hostie de tabarnac), blasphèmes (maudit Christ) et invocations (bonne Sainte-Anne), bien que le français fasse une différence entre ces termes. En général, les jurons leur font pendant en se référant au domaine de la sexualité et de la scatologie. Il est certain que les dérivés sont plus faibles que les sacres, que l'intention de blasphémer ne se trouve plus dans les sacres d'aujourd'hui et que les invocations ont une autre valeur que les composés qui sont plutôt des éléments stylistiques pourvus d'une certaine malléabilité, mais pour nos hypothèses, ces différences sont moins importantes.

Le procès de l'euphémisation joue un rôle crucial dans la distinction entre les sacres et les objets de culte dont ils proviennent. C'est par la prononciation que l'objet liturgique tabernacle devient tabarnac ou, par des procédés dérivationnels, tabarnouche, tabarouette, etc., christ se prononce crisse, calice devient câlice et hostie est parfois réduit jusqu'à la sifflante sti. Il y a donc une double terminologie - religieuse et profane - distinguée par la prononciation et la morphologie des mots. ${ }^{10}$

\section{4 État de la recherche}

Suite à une normalisation de plus en plus visible du français au Québec (Martel \& Cajolet-Laganière 1996, Beauchamp 2004, Lockerbie 2003, Villers 2005) qui va de pair avec la garantie d'une «qualité de la langue » (Cajolet-Laganière \& Martel 1995, Bouchard 2005) et la recherche d'un «bon usage » (Martel 2000), les sacres font certes l'objet de maintes polémiques mais sont aussi traités en tant que termes typiquement québécois. Aujourd'hui, les sacres sont considérés comme une marque d'identification de tout un peuple, non seulement de la classe ouvrière (cf. par exemple Bélanger 2004).

Mais ce n'est qu'à partir de 1937, lors du second congrès de la langue française parlée au Canada que les linguistes accordent aux sacres une attention particulière. L'abbé Henri Raymond condamne l'emploi fréquent de sacres à l'école par les jeunes élèves dont le "langage [était] truffé de jurons et de sacres » (Pichette 1984 : 246). Cette attitude puriste vis-à-vis de la langue persiste jusqu'aux années 1960 et la littérature répressive, que les mandements des évêques illustrent particulièrement bien, est de loin la plus importante. Ce n'est qu'à l'occasion du troisième congrès de 1952 qu'on propose une étude plus systématique de la véritable langue parlée. À partir de ce moment-là, plusieurs chercheurs se mettent à inventorier les sacres utilisés au Québec et à les étudier (dont notamment l'ouvrage de Pichette (1980), commencé en 1968).

L'intérêt que les lecteurs portent aux sacres se reflète dans le grand nombre d'ouvrages de vulgarisation comme celle de Burgen (1998) ou d'Allan \& Burridge (2006) ainsi que de dictionnaires ou glossaires pour touristes ou curieux comme celui de Dulude \& Trait (1991). Il y a aussi un bon nombre de travaux universitaires consacrés aux différents aspects des sacres, comme par exemple ceux de Pichette 1975 et Charette 1999.

En ce qui concerne l'étude scientifique des jurons et expressions vulgaires en général, des recherches sur l'affectivité langagière ont été effectuées dans plusieurs langues comme le démontrent les travaux de Kiener (1983) pour l'allemand notamment, de Jay (1992) pour l'anglo-américain, de Rüsch (1993) pour l'italien, de Ermen (1996) pour le serbo-croate, de Drescher (2003) pour le français de France ou de Kasparian (2005) pour l'acadien. Les recherches effectuées en français québécois décrivent cependant surtout l'évolution des sacres (Tassie 1961, Hardy 1989, Poirier 2006), leurs aspects psychosociaux (Charest 1974), grammaticaux (Gérard 1978, Detges 1993, Léard 1995), fonctionnels (Pichette 1980), sociolinguistiques (Vincent 1982) ou morphologiques et socioculturels (Légaré \& Bougaïeff 1984). Même si quelques-uns de ces travaux reposent sur les corpus de québécois établis dans les années 1970, ils privilégient une approche sociolinguistique, tandis que les études s'appuyant sur l'analyse de conversations se font encore rares. C'est notamment l'étude de Drescher (2000) pour le québécois, tout comme celle de Reisigl (1999) pour l'allemand parlé dans le Tyrol du Sud (Italie), qui reposent sur la pragmatique en général et l'analyse conversationnelle en particulier s'intégrant de fait parfaitement au courant actuel qu'est la linguistique de corpus ${ }^{11}$. C'est pour cela que nous reprendrons et poursuivrons ici les hypothèses avancées par cette linguiste, selon lesquelles les sacres en français québécois, porteurs de sens essentiellement pragmatique et formes plurifonctionnelles, peuvent avoir, outre l'expression 
d'émotions, la fonction de marqueurs discursifs dans le rôle d'une interjection secondaire et qu'ils servent à travers leur emploi à organiser le discours (Drescher $2000: 135-136)$.

Il existe déjà un nombre considérable de travaux sur les caractéristiques pragmatiques du français québécois, mais ce sont les marqueurs discursifs du type et alors?, ben, tu vois, $t$ 'sais (cf. Dostie 2004), par exemple (cf. Vincent 1995) ou encore je veux dire, moi, il dit/je dis/j'ai dit (cf. Vincent 1993) qui y sont discutés. Seul le sacre osti est analysé par Vincent (1993 : 84) dans une approche sociolinguistique.

\section{Enquête sur le terrain et corpus}

\subsection{Enquête sur le terrain et corpus de langue parlée}

Les données empiriques sur lesquelles repose cette étude ont été recueillies au Québec en 2006 et se caractérisent par le choix de lieux, groupes sociaux, registres et situations différents. Le corpus est donc constitué du québécois parlé dans différentes régions de la province (Outaouais, Montréal, Québec, Chaudière-Appalaches) et les locuteurs sont issus aussi bien de zones rurales qu'urbaines. ${ }^{12}$ En vue d'une variation diastratique, les informateurs choisis appartiennent en outre à différentes classes sociales, à différentes catégories d'âge et de sexe, et ont un niveau d'éducation différent.

Mais l'établissement d'un corpus de sacres ne va pas sans difficultés. Dans des situations de conversations surveillées ou dans des contextes plus officiels, les sacres sont peu nombreux, car une norme est imposée par la situation et les locuteurs se trouvent sous contrainte sociale et/ou morale. D'autres situations favorisent cependant l'apparition des sacres qui dépend du milieu, du sujet de la conversation et du langage des locuteurs, d'où la nécessité de prendre en compte la variation diastratique même si le travail ne repose pas sur une analyse sociolinguistique.

Créer une situation favorable aux sacres est problématique. Après une pré-enquête menée avec des Québécois, nous avons intentionnellement renoncé aux entrevues même de type semi-directif, la raison étant que les sacres touchent le phénomène de tabou. De plus, en ce qui concerne la qualité de la langue au Québec, le complexe d'infériorité existe toujours parmi (certains) locuteurs. Par conséquent ceux-ci ont tendance à « améliorer » leur façon de parler, en évitant d'employer des sacres, davantage encore face à des étrangers.

Le « paradoxe de l'observateur » décrit par Labov en 1972 - comment observer les gens parler quand ils ne se sentent pas observés - a été crucial pour la qualité des enregistrements, tandis que tous les informateurs ont très vite perdu leur «peur devant le micro ». C'est la raison pour laquelle nous avons choisi de ne pas être présents lors des enregistrements ou, si notre présence s'avérait nécessaire, de ne pas participer aux conversations. Le corpus contient donc des enregistrements de conversations libres et dans un contexte « naturel» (entre amis, en famille, dans un bar, sur un chantier, etc.), sans cependant perdre de vue les situations plus formelles comme un rendez-vous chez le médecin ou chez la coiffeuse, un cours universitaire, etc. Les enregistrements contiennent toutefois des types discursifs différents tels que des conversations, des monologues ainsi que des situations d'entrevues (mais entre locuteurs natifs).

\subsection{Les sacres dans les médias ${ }^{13}$}

Même si les sacres font partie intégrante de la langue parlée au Québec et que l'on peut les entendre dans la rue, dans le métro, dans les cours d'école ou dans les bistrots, ce n'est pas aussi évident pour les médias. Il est difficile de trouver des sacres dans les émissions de radio et de télévision, car les médias servent aujourd'hui, plus que jamais, d'exemple linguistique ${ }^{14}$. Le Journal de Québec du 20 octobre 2006 cite Radio-Canada, selon laquelle « les jurons, les blasphèmes, les termes grossiers et tout autre propos offensant appartenant aux niveaux de langage vulgaire et très vulgaire peuvent être tolérés dans les dramatiques et les autres textes de création $»$. 
Étant donné notre intérêt pour la langue parlée authentique, nous avons renoncé aux émissions de télévision scriptées ou scénarisées comme les téléromans et téléséries de même que les journaux télévisés. Nous avons au contraire opté pour les émissions de téléréalité comme Loft Story (diffusée sur TQS) ou Occupation double (sur TVA) ou bien les émissions de radio des stations qui s'adressent aux jeunes (par exemple Choi-FM à Québec ou 98.5FM à Montréal) afin d'enregistrer la langue la plus authentique possible. D'autre part, pour voir si les sacres apparaissent dans des contextes plus formels, nous avons inclus des entrevues avec des personnages de la vie publique à la radio et des débats télévisés comme Tout le monde en parle (à Radio-Canada), Les francs-tireurs (à TQc) ou l'émission en direct Il va avoir du $\operatorname{sport}\left(\right.$ à TQc). ${ }^{15}$

\subsection{Les sacres dans la littérature}

Un autre corpus s'ajoute à ceux présentés plus haut, et reposera quant à lui sur la langue écrite tirée de la littérature qui imite la langue parlée, ce que Goetsch (1985) appelle «fingierte Mündlichkeit 》 (oralité fictive). Au Québec, cette littérature est particulièrement abondante dans les années soixante soixante-dix où elle est souvent qualifiée de « littérature joualisante ». La multitude des sacres ne s'interprète d'ailleurs pas comme exagération de la part des auteurs, mais plutôt comme une reproduction fidèle de l'emploi des sacres dans le parler familier québécois de l'époque (cf. Garon 1974 : 3). Ce parler a longtemps été appelé «joual », langage utilisé d'abord par la classe ouvrière de Montréal. Il s'agit d'auteurs comme Jacques Renaud (Le cassé, 1964) ou Michel Tremblay (Les belles-soeurs, représentée pour la première fois en 1968) ou Victor-Lévy Beaulieu (Un rêve québécois, 1972). Une source de documentation très utile se trouve sur le site Web du TLFQ de l'Université Laval : les fichiers lexicaux donnent les sacres dans un contexte littéraire assez large avec la source exacte (auteur, œuvre, année et page).

\section{Hypothèses et analyses des corpus}

\subsection{Hypothèses}

En remplissant différentes fonctions, à savoir grammaticales, émotives et communicatives, les sacres apparaissent le plus souvent sous forme d'interjection (cf. Drescher 2000 : 141).

Les interjections sont une classe grammaticale très hétérogène, car y sont rassemblées toutes les unités difficiles à classifier comme les interjections au sens strict du terme, les onomatopées, les jurons et invectives ainsi que les mots du discours ${ }^{16}$. C'est en effet par leur invariabilité morphologique et leur autonomie syntaxique qu'elles constituent une classe à part. Les linguistes semblent cependant d'accord sur le fait que les interjections sont des mots qui transportent notamment des émotions ou d'autres états subjectifs. Leur fonction principale serait donc la décharge émotive. Dans une perspective formelle, on peut distinguer deux types d'interjections : les interjections primaires et secondaires. Le premier groupe contient des particules illocutoires comme ah, oh, aïe, hein, etc. qui se caractérisent par leur emploi exclusivement interjectif. Le deuxième groupe est plus difficile à délimiter mais il s'agit là d'emplois dérivationnels de mots lexicaux qui ont perdu leur signification première. Cette différenciation remonte à Wundt (1904), reprise par Burger (1980: 56) et plus récemment par Ameka (1992), qui classifie les sacres parmi les interjections secondaires, car celles-ci servent à transmettre de l'affectivité mais ont perdu leur sens premier, ce qui correspond à la définition d'interjection secondaire proposée par Wundt et Burger.

\subsection{Analyses ${ }^{17}$}

L'étude des extraits suivants repose sur l'analyse conversationnelle, méthode élaborée par Harvey Sacks, Emanuel Schegloff et Gail Jefferson dans les années 1970 et poursuivie aujourd'hui par des linguistes comme Auer (2004, 2005) ou Mondada (2002, 2006). Cette méthode d'inspiration ethnométhodologique $^{18}$ se caractérise par l'étude minutieuse de ce qui se produit dans les interactions 
verbales. Les analyses de conversation s'intéressent en particulier à l'organisation séquentielle des tours de parole. Pour ce faire, les locuteurs ont recours à des modèles langagiers institutionnalisés - modèles que l'analyse conversationnelle se propose d'étudier.

$\mathrm{Du}$ point de vue de l'analyse conversationnelle, on peut s'attendre au fait que les sacres se laissent catégoriser, à savoir qu'ils peuvent remplir des fonctions particulières au sein de l'organisation de la conversation. Comme nous l'avons évoqué précédemment, la catégorisation proposée par Drescher $(2000)^{19}$ nous paraît toujours pertinente quoique ses données datent des années 1970. Un premier balayage de notre corpus établi en 2006 donne les mêmes fonctions de sacres interjectifs, mais en révèle également d'autres.

\subsubsection{L'intensification d'un énoncé à portée affective, évaluative ou subjective}

(1) Le couple $\mathrm{R}$ et $\mathrm{D}$ a invité un autre couple ( $\mathrm{H}$ et $\mathrm{F}$ ) et ils sont assis autour de la table. Les deux hommes sont en train de discuter du patron de $\mathrm{H}$ qui a agrandi sa propriété. [ChezLesLSacreur011106Seigneur]

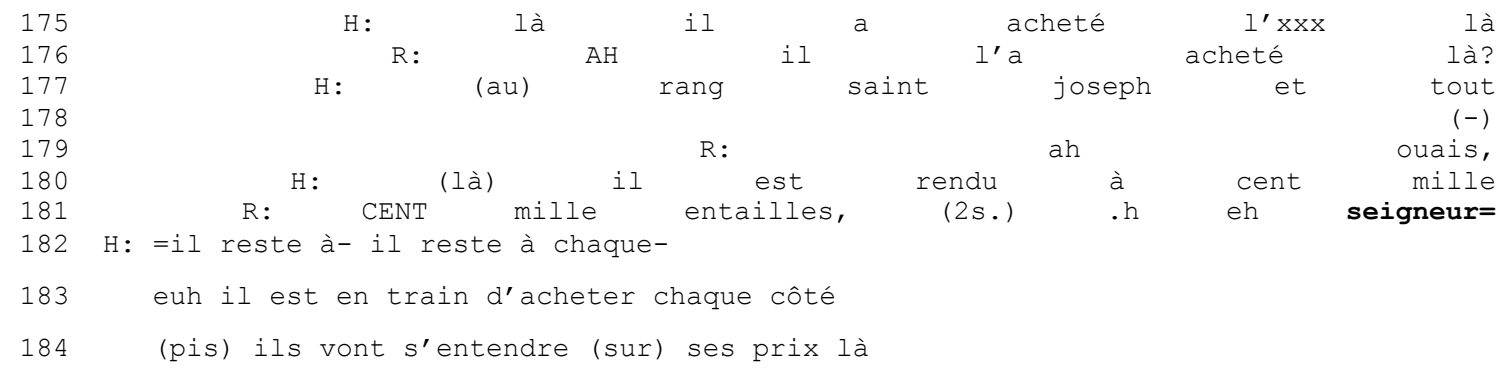

(2) Grignon, Claude-Henri (1894-1976). Homme. 1933, p. 201. [Fichiers lexicaux, TLFQ; littérature].

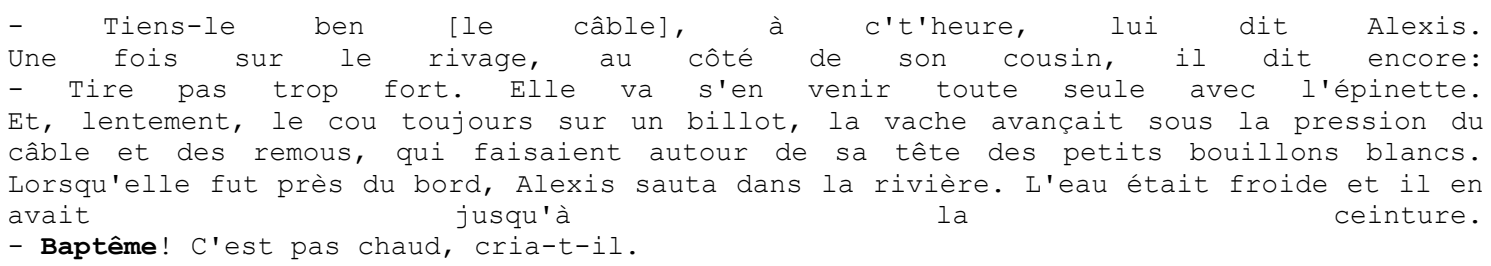

(3) Les présentateurs A et D parlent du lancement du calendrier Dream team 007 qui a eu lieu la veille de cette émission. [cassette 081106, face A]

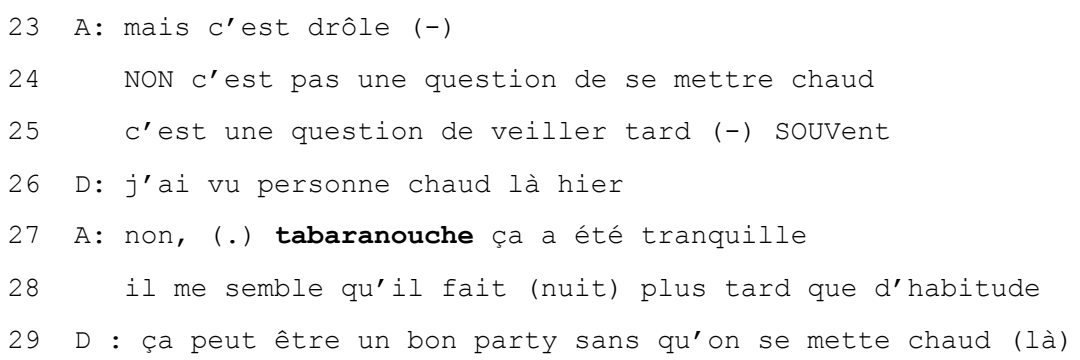

Dans l'exemple (1), le sacre est précédé d'une interjection primaire (« eh »). Par rapport à l'énonciation à laquelle les sacres se réfèrent dans tous les trois extraits («cent mille entailles » (1), «c'est pas chaud» (2), «ça a été tranquille»(3)), ils peuvent être soit postposés soit antéposés. En outre, ils peuvent souligner une énonciation soit plutôt positive (exemples 1 et 3) soit plutôt négative (exemple 2). Mais les sacres n'expriment jamais cette évaluation positive ou négative, ils l'intensifient seulement. Ce sont uniquement les énonciations qui expriment une évaluation. 


\subsubsection{Le signal d'écoute à valeur expressive}

(4) Les amis sont assis autour d'une table. Un homme explique les incidents récents qui ont eu lieu dans la ligue nationale de hockey. [FillesTablesMH181106MonDoux]

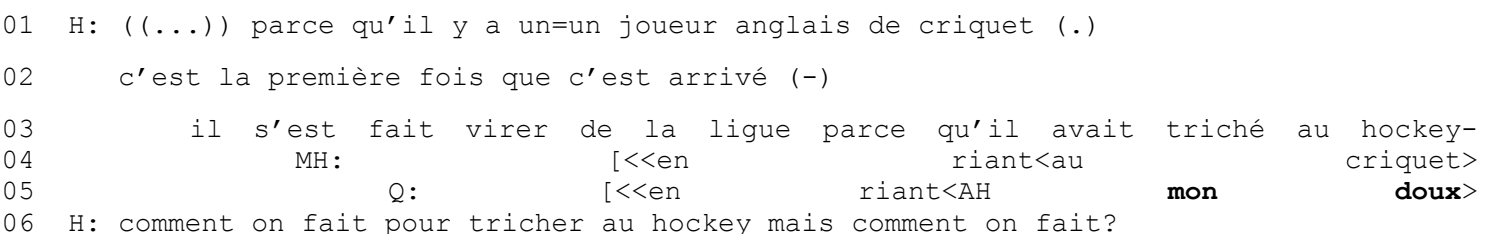

(5) Le présentateur $\mathrm{A}$ fait une devinette à son équipe. Il s'agit du 9 novembre dans l'histoire. [cassette 071106, face B]

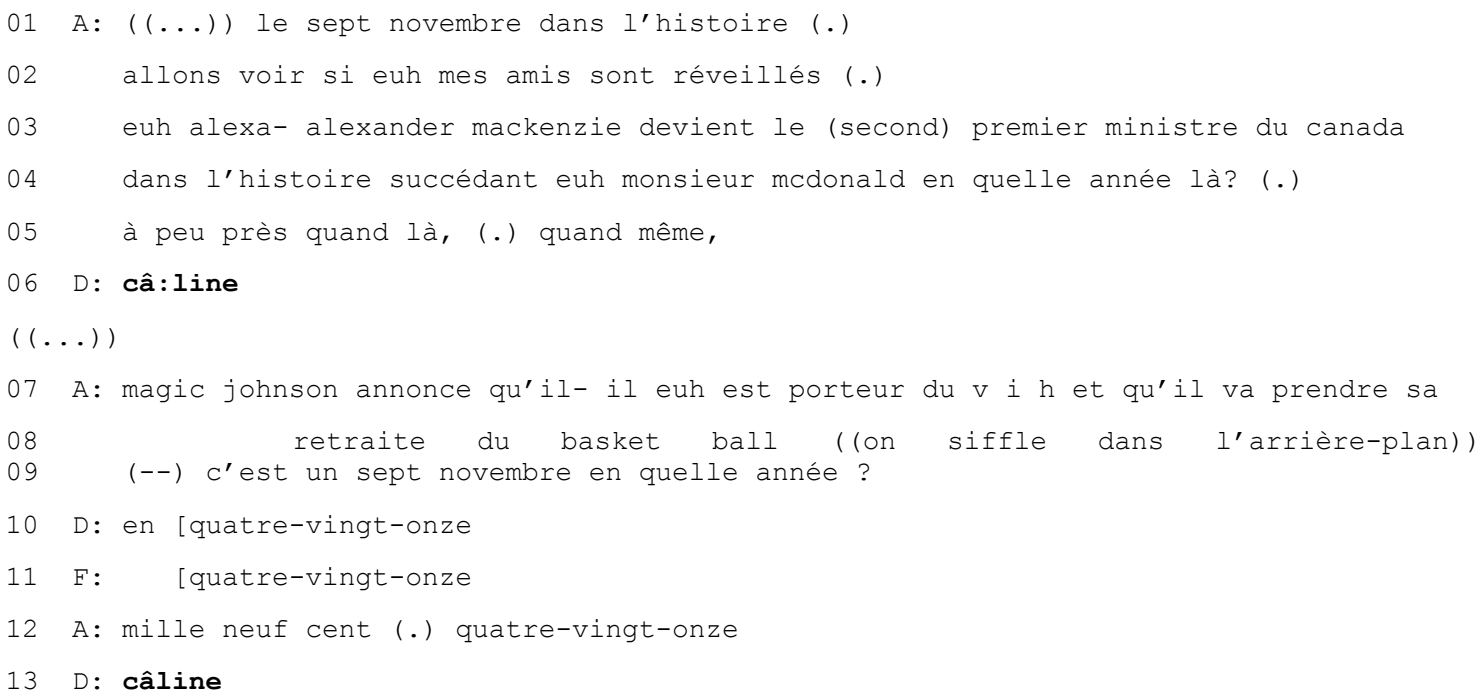

Les signaux d'écoute, ou le back channel pour reprendre le terme utilisé par Laforest (1992), servent à commenter les propos d'un locuteur. Ils représentent le plus souvent un tour de parole à part entière, mais ils peuvent être accompagnés d'un rire (cf. l'exemple 4), d'une toux ou d'une aspiration qui renforcent la surprise normalement exprimée par ces signaux. En outre, par ces commentaires, les autres locuteurs assurent à celui ou celle qui parle qu'ils l'écoutent, et c'est une façon pour eux de participer à la conversation sans réclamer le tour de parole.

\subsubsection{L'interruption}

(6) L'étudiant $\mathrm{P}$ fait régulièrement du cardio au gymnase de l'université et en parle à son ami G. [GarsUdM211106Bâtard]

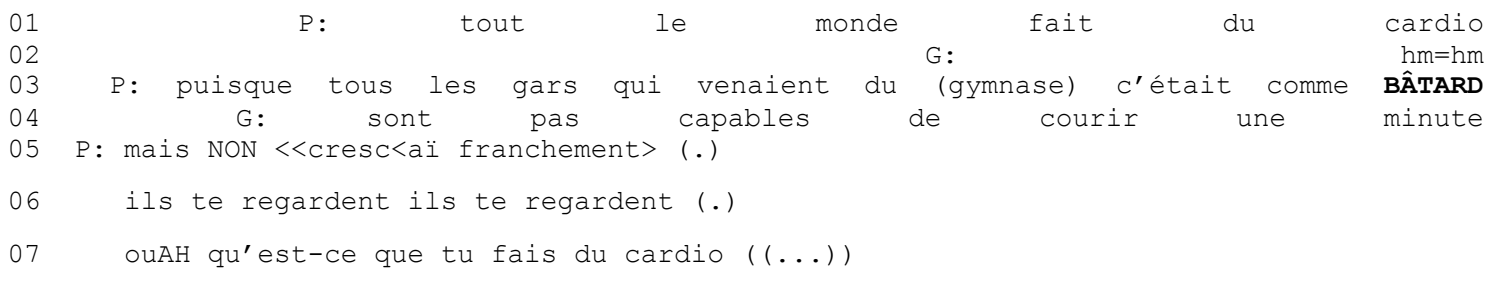

(7) Les amis F, H - maintenant à la retraite - et $\mathrm{V}$ parlent de l'ancien travail de $\mathrm{H}$. [AncienMaire261106Calvaire] 
01 F: peut-être que ça (vous) donne l'hiver de (la) vie

$02 \mathrm{H}: \mathrm{C}^{\prime}$ est ça=

$03 \mathrm{~F}:($ (rire))

$04 \mathrm{H}$ : =non mais C'est vrai là sans farce là (-)

05 c'est comme (si) xxx les hivers étaient plus xxx de ma vie

06 F: la chaleur

$07 \mathrm{~V}$ : oui

$08 \mathrm{H}$ : t'as la chaleur tout le temps là pis (-) calvaire

09 V: c'est pas (-) (pas) de res[ponsabilité

$10 \mathrm{H}:$

[pas de responsabilité

Dans ces exemples, les sacres marquent la fin d'un tour de parole qui reste cependant incomplet d'un point de vue syntaxique. Cette interruption dans le tour de parole donne - dans tous les trois exemples le droit de parole à l'autre locuteur. Les sacres mis à la fin d'un tour de parole correspondent ici à une pause, une hésitation avant de savoir comment continuer la phrase. Étant donné qu'ils sont précédés par « comme » (exemple 6), les sacres 'résument' ou remplacent la partie du tour de parole qui n'est plus exprimée. Il en est de même dans l'exemple (7) où le tour de parole est interrompu par le mot «pis », suivi d'abord d'une pause, puis du sacre «calvaire». Ils remplacent une interjection primaire comme « euh », quoiqu'il s'agisse, contrairement aux interjections primaires, d'un élément évaluatif inhérent. Ces 'mots-charnières' donnent aux autres locuteurs la possibilité de reprendre la parole.

\subsubsection{L'autocitation ${ }^{20}$}

(8) Conversation à propos de la petite cousine de C (âgée d'un an) qui fait caca dans l'eau quand elle prend le bain, ce que son frère, âgé de 4 ans, n'aime pas du tout. [Réunion091006Tabarnouche]

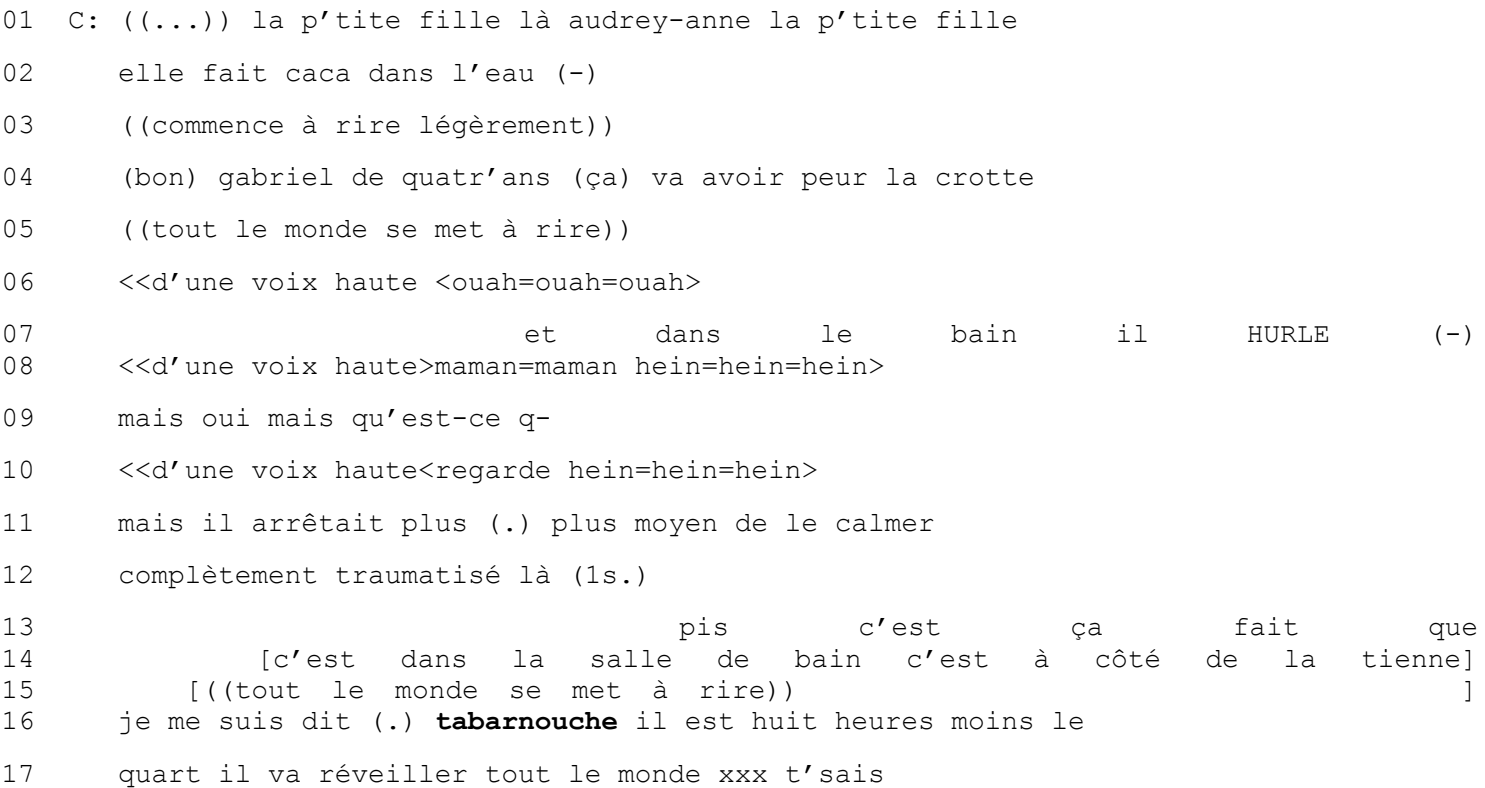

(9) Nantel, Adolphe (1886-1954): Hache. 1932, p. 131-132. [Fichiers lexicaux, TLFQ ; littérature].

- C'est rien que de l'ouvrage comme ane autre, ça... J'ai déjà vu pire... quand j'ai passé sous les billots, y a 12 ans, aux Chutes des Cinq, dans la rivière Mattawin... Y en avait ben un mille de cordé... J'veux donner la chance à un jeune de se sauver, la «jam» avance d'un pied, je saute, et v'lan dans l'rapide. J'sus sorti tout nu en bas d'la chute. C'était plus risqué qu'icite. J'dois vous dire que j'avais eu moins chaud... 
Les éléments introducteurs d'une autocitation sont les verbes de communication (verba dicendi) - dans les exemples, il s'agit de « je me suis dit» (8) et «j'leur crie » (9). Après cette formule métalinguistique, les sacres marquent le début d'une autocitation de type «monologue intérieur» (exemple 8) ou de type exclamatoire (exemple 9). Par le choix de cette stratégie, les locuteurs arrivent à rendre plus présentes et plus animées les pensées qui les occupaient au moment des faits ou bien à mieux rendre leurs propos lorsque survint l'évènement. Dans les extraits transcrits, les sacres marquant le début de l'autocitation sont précédés par une petite pause, élément de la langue parlée normalement remplacé dans la langue écrite par les deux-points (cf. exemple 9). En outre, la partie citée se distingue nettement de la partie citante par l'intonation qui change avec les sacres et se maintient ainsi jusqu'à la fin de ce segment.

On peut d'abord constater que les sacres comme interjections secondaires accompagnent et intensifient souvent des énoncés et sont soit postposés soit antéposés. Ensuite, ils n'apparaissent que rarement isolés, mais le plus souvent en relation avec soit une interjection primaire précédant ou suivant les sacres soit un signal non-verbal (aspiration, rire, etc.). Par conséquent, ces exemples ont montré qu'à part leurs fonctions grammaticales et émotives, les sacres peuvent remplir des fonctions interactionnelles comme celle de marqueurs discursifs.

\section{Conclusion et perspectives}

Au cours de cette analyse nous avons tenté de montrer que les sacres en français québécois actuel représentent beaucoup plus que de simples jurons à base affective, servant uniquement à se défouler verbalement, et sont donc loin d'être des tics de langage, des locutions de remplissage ou de simples interjections introduites un peu partout à l'intérieur d'un énoncé. Ils s'avèrent être plutôt des termes plurifonctionnels remplissant non seulement différentes fonctions grammaticales mais aussi interactionnelles dans l'organisation du discours.

On retrouve à peu près les mêmes emplois que les Québécois font de leurs sacres dans tous les genres discursifs étudiés, à savoir les conversations quotidiennes, à la radio ou à la télévision ainsi que dans la littérature qui imite la langue parlée.

Outre les analyses effectuées ci-dessus, nous avançons l'hypothèse selon laquelle les tours de parole contenant les sacres (le plus souvent une interjection primaire, un sacre comme interjection secondaire et un énoncé évaluatif) forment une courbe d'intonation caractéristique servant ainsi - une fois de plus - à la structuration du discours. ${ }^{21}$

À travers les sacres qui représentent un aspect caractéristique, largement discuté du français québécois, nous avons vu que cette "québécité " ${ }^{22}$ peut être analysée dans une nouvelle perspective, en procédant à une analyse conversationnelle, car celle-ci révèle en effet - indépendamment du discours puriste - leur fonction interactionnelle de marqueurs discursifs dans l'organisation de la conversation.

\section{Références bibliographiques}

Allan, K./Burridge, K. (2006). Forbidden Words. Taboo and the Censoring of Language. Cambridge: Cambridge Univ. Press.

Ameka, F. K. (1992). Interjections: the universal yet neglected part of speech. Journal of Pragmatics, 15, 492-498.

Auer, P./Rönfeldt, B. (2004). Prolixity as adaption prosody and turn-taking in German conversation with a fluent aphasic. In Couper-Kuhlen, E. et al. (éd.), Sound patterns in interaction. Amsterdam: Benjamins, 171-200.

Auer, P. (2005). Projection in Interaction and Projection in Grammar. Text. An Interdisciplinary Journal for the Study of Discourse, 25 (1), 7-36. 
Authier-Revuz, J. (1984). Hétérogénéité(s) énonciativé(s). Langages, 73, 98-111.

Beauchamp, L. (2004). Le français standard en usage au Québec. (Allocution de la ministre de la culture et des communications, madame Line Beauchamp, 17.02.04, Université de Sherbrooke). $<$ http://www.spl.gouv.qc.ca/secretariat/d_subvention_Franqus.html> (consulté le 13.03.07).

Beauchemin, N./Martel, P. et al. (1981). Échantillon de textes libres no VI. Sherbrooke : [université].

Bélanger, M. ( $\left.{ }^{2} 2004\right)$. Petit guide du parler québécois. Outremont : Stanké.

Benveniste, E. (1974). La blasphémie et l'euphémie. In Benveniste, E., Problèmes de linguistique générale. Vol. 2. Paris : Gallimard, 254-257.

Bouchard, C. (2005). La question de la qualité de la langue aujourd'hui. In Stefanescu, A./Georgeault, P. (éds), Le français au Québec. Les nouveaux défis. Montréal : Fides, 387-97.

Bovet, L. (1977). Le vocabulaire du défoulement au Québec ou Petite enquête sur les sacres et les jurons. Langues et linguistique, 3, 27-46.

Burgen, S. (1998). Bloody hell, verdammt noch mal! Eine europäische Schimpfkunde. München: Dt.Taschenbuchverlag.

Burger, H. (1980). Interjektionen. In Sitta, H. (éd.), Ansätze zu einer pragmatischen Sprachgeschichte. Tübingen: Niemeyer, 53-69.

Cajolet-Laganière, H./Martel, P. (1993). Entre le complexe d'infériorité linguistique et le désir d'affirmation des Québécois et Québécoises. Cahiers de l'Institut de linguistique de Louvain (CILL), 19/3-4, 169-185.

Cajolet-Laganière, H./Martel, P. (1995). La qualité de la langue au Québec. Québec: Institut Québécois de Recherche sur la Culture.

Charest, G. (1974). Le livre des sacres et blasphèmes québécois. Montréal : L’Aurore.

Charette, É. (1999). Du sacre au nom de qualité: approche théorique et description grammaticale (mémoire de maîtrise, Université de Sherbrooke). Sherbrooke : [université].

Coulon, A. (2002). L'Ethnométhodologie. Paris : PUF (= Que sais-je?; 2393).

Detges, U. (1993). Être en sacre. Funktionsverbgefüge und ihre delokutive Derivation im Français Québécois. Neue Romania, 14, 63-90.

Dostie, G. (2004). Pragmaticalisation et marqueurs discursifs. Analyse sémantique et traitement lexicographique. Bruxelles : De Boeck, Duculot.

Drescher, M. (2000). Eh tabarnouche! C'était bon. Pour une approche communicative des jurons en français québécois. Cahiers de praxématique, 34, 133-160.

Drescher, M. (2003). Sprachliche Affektivität. Darstellung emotionaler Beteiligung am Beispiel von Gesprächen aus dem Französischen. Tübingen: Niemeyer.

Ducrot, O. (1984). Polyphonie. In Ducrot, O., Le dire et le dit. Paris : Minuit, 171-233.

Dulong, G. (1984). L'Atlas linguistique de l'Est du Canadas. In Amyot, M. (éd.), Le statut culturel du français au Québec: Actes du congrès Langue et Société au Québec à Québec 1982. Vol. 2. Québec : Le Conseil de la Langue Française, 188-194.

Dulude, Y./Trait, J.-C. (1991). Dictionnaire des injures québécoises. Québec : Stanké.

Ermen, I. (1996). Fluch - Abwehr - Beschimpfung. Pragmatik der formelhaften verbalen Aggression im Serbokroatischen. Bern, Berlin et al.: Lang.

Filion, M. (1984). Religion et langue. In Amyot, M. (éd.), Le statut culturel du français au Québec: Actes du congrès Langue et Société au Québec à Québec 1982. Vol. 2. Québec : Le Conseil de la Langue Française, 107-112.

Gagnon, S. (2000). La langue, gardienne de la foi. In Plourde, M. (éd.), Le français au Québec, 400 ans d'histoire et de vie. Montréal: Fides/Publications du Québec, 171-175.

Garfinkel, H. (1967). Studies in ethnomethodology. Englewood Cliffs, N.J. : Prentice-Hall. 
Garon, C. H. (1974). Le sacre dans les oeuvres de Michel Tremblay et de Roch Carrier. [S.1.] (thèse McMaster University, 1973).

Gérard, J. (1978). Mon ostie de... Cahier de linguistique, 8, 163-179.

Goetsch, P. (1985). Fingierte Mündlichkeit in der Erzählkunst entwickelter Schriftkulturen. Poetica, 17, 202-218.

Goffman, E. (1980). Rahmen-Analyse. Ein Versuch über die Organisation von Alltagserfahrungen. Frankfurt a. M.: Suhrkamp.

Govaert-Gauthier, S. (1979). Attitudes de vingt-huit Montréalais francophones sur le français parlé au Québec. In Thibault, P. (éd.), Le français parlé. Etudes sociolinguistiques. Edmonton : Linguistic Research, 145-152.

Grevisse, M./Goosse, A. $\left({ }^{13} 2001\right)$. Le bon usage : grammaire française. Paris et al.: Duculot.

Hardy, R. (1989). Ce que sacrer veut dire: à l'origine du juron religieux au Québec. In Delumeau, J. (éd.), Injures et blasphèmes. Paris : Imago, 99-125.

Hewson, J. (2000). The French Language in Canada. München: LINCOM Europa.

Jay, T. (1992). Cursing in America. A psycholinguistic study of dirty language in the courts, in the movies, in the schoolyards and on the streets. Philadelphia: Benjamins.

Kasparian, S. (2005). Gros mots et euphémismes linguistiques en acadien. In Brasseur, P./Falkert, A. (éds), Français d'Amérique: approches morphosyntaxiques. Paris: L'Harmattan, 313-326.

Kiener, F. (1983). Das Wort als Waffe. Zur Psychologie der verbalen Aggression. Göttingen: Vandenhoeck \& Ruprecht.

Labov, W. (1972). Sociolinguistic patterns. Oxford: Blackwell, 61-62, 209.

Laforest, M. (1992). Le back-channel en situation d'entrevue. Québec : CIRAL, Université Laval.

Laforest, M. (2002). Attitudes, préjugés et opinions sur la langue. In Verreault, C. (éd.), Le français, une langue à apprivoiser. Québec : Presses de l’Université Laval, 81-91.

Landry, L. (1972). Québec français ou Québec québécois ? Montréal : Presses libres.

Laurendeau, P. (2007). Avoir un méchant langage. Du comportement social dans les représentations épilinguistiques de la culture vernaculaire : le cas du Québec francophone. Glottopol, 9, 22-49.

Léard, J.-M. (1995). Grammaire québécoise d'aujourd'hui. Comprendre les québécismes. Montréal : Guérin.

Légaré, C./Bougaïeff, A. (1984). L'empire du sacre québécois. Etude sémiolinguistique d'un intensif populaire. Québec : Presses de l'Univ. du Québec.

Lockerbie, I. (2003). Le débat sur l'aménagement du français au Québec. Globe. Revue internationale d'études québécoises, 2/2, 125-149.

Martel, P./Cajolet-Laganière, H. (1996). Le français québécois. Usages, standard et aménagement. Québec : Institut Québécois de Recherche sur la Culture.

Martel, P. (2000). Le Bon usage au Québec. In Schafroth, E. (éd.), Französische Sprache und Kultur in Quebec. Hagen: ISL-Verlag, 11-40.

Mondada, L. (2002). Pour une approche interactionnelle de la catégorisation des ressources linguistiques par les locuteurs. Cahiers de l'institut de linguistique de Louvain, 28 (3/4), 23-35.

Mondada, L. (2006). Participants' online analysis and multimodal practices: projecting the end of the turn and the closing of the sequence. Discourse studies, 8/1, 117-131.

Montagu, A. (1967). The anathomy of swearing. New York : The Macmillan Company.

Pichette, J.-P. (1975). Les jurons au Canada français : étude de l'histoire de la langue et de la littérature orale suivie du recueil des jurons (mémoire de maîtrise, Université Laval). Ste-Foy : [université].

Pichette, J.-P. (1980). Le guide raisonné des jurons. Montréal : Quinze. 
Pichette, J.-P. (1984). Jurons franco-canadiens : typologie et évolution. In Amyot, M. (éd.), Le statut culturel du français au Québec: Actes du congrès Langue et société au Québec. Vol. 2. Québec: Le Conseil de la Langue Française, 245-272.

Poirier, C. (2006). Le sacre est-il proprement québécois? Québec français, 143, 23-24.

Pusch, C. D./Raible, W. (éds). (2002). Romanistische Korpuslinguistik. Korpora und gesprochene Sprache. Tübingen: Narr.

Radio-Canada. (2006). La politique de Radio-Canada. Le Journal de Québec 20.10.06, 46.

Raymond, D./Lafrance, A. A. (2001). Norme et médias. Québec : Les publications du Québec (= Terminogramme ; 97-98).

Reisigl, M. (1999). Sekundäre Interjektionen. Eine diskursanalytische Annäherung. Frankfurt/Main: Lang.

Riegel, M./Pellat, J.-C. et al. (1994). Grammaire méthodique du français. Paris : PUF.

Robinson, S./Smith, D. (1984). Practical Handbook of Quebec and Acadian French / Manuel pratique du français québécois et acadien. Toronto et al.: Anasis.

Rüsch, P. (1963). Invokations- und Fluchformeln im Italienischen. Winterthur: Keller.

Selting, M. et al. (1998). Gesprächsanalytisches Transkriptionssystem (GAT). Linguistische Berichte, 173, 91-122.

Stefanescu, A./Georgeault, P. (éds). (2005). Le français au Québec : les nouveaux défis. Montréal : Fides.

Tassie, J.-S. (1961). The Use of Sacrilege in the Speech of French Canada. American Speech, 36, 34-40.

Thibault, J./Vincent, D. (1981). Le sacre en français montréalais : Aspects fonctionnels et dynamique expressive : Quand les intensificateurs phatiquent. Le français moderne, 49/3, 206-215.

Tremblay, M. (1980). Thérèse et Pierrette à l'école des Saints-Anges. Montréal : Leméac.

Trésor de la langue française au Québec : fichier lexical. Université Laval. <http://www.tlfq.ulaval.ca/fichier/> (consulté le 07.03.07)

Villers, M.-E. (2005). La norme réelle du français. In Stefanescu, A./Georgeault, P. (éds), Le français au Québec. Les nouveaux défis. Montréal : Fides, 399-420.

Vincent, D. (1981a). Norme, langage expressif et sacre en français montréalais. 'C'est pas beau mais ça défoule'. In Klinkenberg, J.-M./Racelle-Latin, D. et al. (éds), Langages et collectivités : le cas du Québec. Actes du Colloque de Liège, 25-28 mars 1980. Montréal : Leméac.

Vincent, D. (1981b). A quoi servent les mots qui ne servent à rien. Culture, I (2), 61-65.

Vincent, D. (1982). Pressions et impressions sur les sacres au Québec. Québec : Office de la Langue Française.

Vincent, D. (1993). Les ponctuants de la langue et autres mots du discours. Québec : Nuit blanche.

Vincent, D. (1995). Remarques sur par exemple en français québécois. Le Français moderne, 63/1, 55-71.

Wundt, W. (1904). Völkerpsychologie. Vol. 1, 1. Leipzig: Philo Verlagsges.

\footnotetext{
${ }^{1}$ Voir par exemple Bovet (1977), Vincent (1981a) et Hewson (2000).

${ }^{2} \mathrm{Au}$ Québec, la terminologie courante pour cet équivalent de France est « sacre » (cf. 2).

${ }^{3}$ On a longtemps reproché aux Québécois de mettre les sacres là où le bon mot leur échappe, et c'est entre autres pour cela que le sujet de l'insécurité linguistique et la perception de la langue chez les Québécois a fait couler beaucoup d'encre (cf. par exemple Govaert-Gauthier 1979, Cajolet-Laganière \& Martel 1993, Laforest 2002, Laurendeau 2007).

${ }^{4}$ Cf. l'ouvrage de référence de Montagu (1967).

${ }^{5} \mathrm{Cf}$. pour le choix terminologique plus détaillé sous 3 .

${ }^{6}$ Exemples (cf. Hewson 2000 : 63s.) : «Le câliss me frappe en plein dans la jambe (...).» (câliss = substantif); «Avant que je réalise ce qui se passe, (...), le baril me crisse en l'air d'une shotte. » (crisse = verbe); «Fa' que j’me
} 
suis tenu après la corde en ostie! » (en ostie = adverbe 'beaucoup') ; «A moitié chemin descendant, je rencontre-tu pas le tabarnac de baril qui monte asteur! » (tabarnac = adjectif).

${ }^{7}$ Il s'agit de la grammaire de Léard (1995).

${ }^{8}$ Les auteurs font surtout la distinction entre sacre, juron et blasphème (cf. par exemple Charest 1974 ou Pichette 1984), et c'est la terminologie de Vincent (1982 : 35ss.) qui nous paraît la plus détaillée : juron, sacre, blasphème, invocation, dérivé, composé.

${ }^{9}$ L'effet des sacres s'est perceptiblement atténué avec la perte d'influence de l'église catholique dans la vie publique au Québec depuis la Révolution tranquille des années 1960 (cf. par exemple les commentaires à propos de la relation entre la langue et la religion/foi de Filion (1984) ou de Gagnon (2000). C'est d'ailleurs très probablement pour cela que l'on observe un processus de pragmaticalisation, au moins pour les jeunes. C'est-à-dire le tabou religieux qu'étaient les sacres est plutôt devenu un tabou social : ces mots-là ne se disent pas parce que ce n'est pas beau. Dans plusieurs analyses, Vincent (1981b, 1993) et Thibault \& Vincent (1981) observent que notamment le sacre hostie (souvent réduit à sti) a perdu - chez certains locuteurs - sa valeur exclamative et sa force expressive. Surutilisé par quelques-uns, il est donc devenu phatique ou un «ponctuant » dans la terminologie de Vincent (1993).

${ }^{10}$ Pour les différentes formations et classifications des sacres, cf. notamment Charest 1974, Pichette 1980, Vincent 1982 ou Légaré \& Bougaïeff 1984.

${ }^{11}$ Cf. par exemple le recueil de Pusch \& Raible (2002) avec 37 contributions qui ont pour sujet différentes méthodes de linguistique de corpus ainsi que la présentation de différents projets actuels.

${ }^{12}$ Il nous a semblé intéressant de tenter de répertorier des sacres préférés selon les régions même si, dans le cas du Québec, les chercheurs sont d'accord aujourd'hui que la variation diatopique n'existe que de façon réduite (cf. par exemple Dulong 1984: 189).

${ }^{13}$ Nous tenons à remercier le Centre d'études sur les médias de l'Université Laval qui nous a renseignées sur les différentes émissions.

${ }^{14}$ Cf. par exemple l'ouvrage de Stefanescu \& Georgeault 2005, dans laquelle plusieurs articles traitent la qualité de la langue dans les médias, ou bien celui de Raymond \& Lafrance 2001, numéro entièrement consacré au sujet «norme et médias ».

${ }^{15}$ Il ne faut cependant pas oublier que la plupart des émissions sont toutefois le fruit d'un montage où les sacres sont mis ou omis, donc censuré intentionnellement. En outre, nous avons dû renoncer aux entrevues avec des sportifs, car ceux-ci reçoivent aujourd'hui une formation afin de bien s'exprimer au micro.

${ }^{16}$ Cf. par exemple les grammaires françaises de Grevisse (2001 : 1567s.) ou de Riegel \& Pellat et al. (1994 : 462 464).

${ }^{17}$ Suivant les conventions de transcription actuelles pratiquées par la plupart des universités germanophones, les données empiriques sont transcrites à l'aide du logiciel Praat, logiciel élaboré par l'Institut des sciences phonétiques de l'Université d'Amsterdam, ainsi qu'à l'aide du système GAT, système de transcription proposé par Selting et al. (1998) : [ ] chevauchement, = enchaînement rapide, \& continuation du même tour de parole, (.) micropause, (-), (--), $(--)$ pauses brève, moyenne, longue, $(x \mathrm{~s})$ pause de $\mathrm{x}$ secondes, $\because:$ allongement syllabique, $h m=h m$ signes disyllabiques, be: $n$ allongement d'une syllabe, liEUX accentuation d'un mot, là' accentuation montante, ? intonation montante, , intonation montante moyenne, intonation constante, ; intonation descendante moyenne, $\langle<$ dim $\rangle>$ diminution du volume, $\langle<p>>$ doucement, $<<$ rire $>>$ extension du phénomène décrit, $h$ aspiration, (passage) transcription incertaine, $x x x$ énoncé incompréhensible, $p o$ - interruption perceptible de l'énoncé, $((\ldots))$ omission.

${ }^{18}$ L'ethnométhodologie, quant à elle, s'est développée dans les années 1960 dans la sociologie américaine (Garfinkel 1967), et son objectif consiste à analyser des méthodes utilisées par les membres d'une communauté pour accomplir leurs activités quotidiennes (cf. Coulon 2002: 24).

${ }^{19}$ Son étude repose sur un petit corpus de conversations en québécois parlé dans la région de Sherbrooke recueilli en 1972/73 (Beauchemin \& Martel et al. 1981) ainsi que sur un roman de Michel Tremblay (Thérèse et Pierrette à l'école de Saints-Anges, 1980).

${ }^{20}$ Nombreuses sont les études sur l'autocitation et plus généralement sur l'hétérogénéité énonciative. Drescher (2006 : 10) constate que selon la discipline et le cadre théorique des travaux, les auteurs parlent de "polyphonie » (Ducrot), « hétérogénéité énonciative » (Authier-Revuz) ou «participation framework »(Goffman). 
ISBN 978-2-7598-0358-3, Paris, 2008, Institut de Linguistique Française

Discours, pragmatique et interaction

DOI $10.1051 / \mathrm{cmlf0} 162$

${ }^{21}$ Cette hypothèse repose sur la base de l'analyse prosodique d'une partie de notre corpus à l'aide du logiciel Praat, mais elle demande davantage d'exemples pour être vérifiée ou éventuellement réfutée. Ces exemples proviendront également de notre corpus.

${ }^{22}$ Ce terme figure pour la première fois dans l'ouvrage de Louis Landry intitulé Québec français ou Québec québécois paru en 1972. 